\title{
Functional outcome of patients with prolonged hypoglycemic encephalopathy
}

\author{
Guillaume Barbara', Bruno Mégarbane², Laurent Argaud ${ }^{3}$, Guillaume Louis ${ }^{4}$, Nicolas Lerolle ${ }^{5}$, \\ Francis Schneider ${ }^{6}$, Stéphane Gaudry ${ }^{7,8}$, Nicolas Barbarot ${ }^{9}$, Angéline Jamet $^{10}$, Hervé Outin $^{11}$, Sébastien Gibot ${ }^{1}$ \\ and Pierre-Edouard Bollaert ${ }^{*}$
}

\begin{abstract}
Background: Little is known about the causes, clinical course and long-term outcome of comatose patients with prolonged hypoglycemic encephalopathy.

Methods: In a multicenter retrospective study conducted in patients hospitalized from July 1, 2004, to July 1, 2014, we investigated functional long-term prognosis and identified prognosis factors of patients admitted in an intensive care unit (ICU) with prolonged neurological manifestations related to hypoglycemia. Eligible patients were adults admitted to the ICU with a Glasgow Coma Score $<8$ due to hypoglycemia and persistent consciousness disorders after normalizing blood glucose levels. Patients with possible other causes of consciousness disorders, previous cognitive disorders, hypothermia $<35^{\circ} \mathrm{C}$ or circulatory arrest within $24 \mathrm{~h}$ after ICU admission, were excluded. Follow-up phone call was used to determine patients' functional outcome using modified Rankin Scale (mRS) at a minimum of 1-year follow-up with mRS 0-3 defining good and mRS 4-6 poor outcomes.

Results: Forty-nine patients were included. Causes of hypoglycemia were various, mainly including insulin or oral antidiabetic drugs abuse (65\%) and neuroendocrine carcinoma (16\%). Twenty (41\%) patients died in the ICU, two (4\%) patients further died and nine (18\%) patients had a poor outcome at long-term follow-up. Five patients discharged from the ICU with mRS > 3 improved enough to be in the good outcome group 1 year later. Twenty-two (45\%) patients underwent therapeutic limitation, mainly related to no expected hope for improvement. On multivariate analysis, only low mRS prior to ICU admission (OR 2.6; 95\% Cl 1.1-6.3; $P=0.03$ ) and normal brain imaging (OR 7.1; 95\% $\mathrm{Cl} 1.1-44 ; P=0.03)$ were significantly predictive of a good outcome. All patients $(n=15)$ who remained hypoglycemic $>480$ min had a poor outcome.
\end{abstract}

Conclusion: Poor outcome was observed in about $60 \%$ of this population of hypoglycemic encephalopathy. However, some patients can recover satisfactorily over time.

Keywords: Hypoglycemia, Hypoglycemic encephalopathy, Patient outcome assessment, Intensive care units, Care withdrawal, Brain imaging

\section{Background}

Hypoglycemia is a common disorder, especially observed in diabetic patients. Neurological manifestations of hypoglycemia are wide and nonspecific including confusion, seizures, focal neurological deficits, stupor and

\footnotetext{
*Correspondence: pe.bollaert@chru-nancy.fr

1 Service de Réanimation médicale, Hôpital Central, CHU de Nancy, Av. de Lattre de Tassigny, 54035 Nancy Cedex, France

Full list of author information is available at the end of the article
}

sometimes coma [1-3]. Neurological deficits are usually fully reversible and non-life-threatening in case of short-duration hypoglycemia [4]. Although currently illdefined, the hypoglycemic encephalopathy, which occurs during deep and/or prolonged hypoglycemia, is a sustained comatose state. Seizures and other neurological deficits may also be observed. The short- and long-term evolution of hypoglycemic encephalopathy, from full recovery to persistent vegetative state, remains yet poorly understood [1, 2, 5]. Several studies tried to identify 
prognosis factors but were limited by small sample size and/or short follow-up period $[1,2]$. The hypoglycemic encephalopathy has been demonstrated to induce early lesions of the internal capsule that may secondarily reach the white matter [6,7]. Their prognostic value is debated owing to the variability of the lesions [7] and the absence of correlation between severity of imaging lesions and patient prognosis [1, 6-8]. The objective of this multicenter study was to study causes, initial consequences and the vital and functional long-term prognosis of patients admitted to the ICU with prolonged neurological manifestations related to obvious hypoglycemia.

\section{Methods}

\section{Study participants}

We evaluated the data of patients admitted and treated in the ICU from July 1, 2004, to July 1, 2014, for prolonged hypoglycemic encephalopathy. Fifteen French adult ICUs were invited to participate in the study in September 2015. At each participating ICU, one investigator from the medical staff was responsible for identifying the patients using either administrative PMSI French database or local electronic medical records or both, collecting their data, and contacting them for follow-up news at least 1 year after the ICU admission. The diagnosis of prolonged hypoglycemic encephalopathy was considered in all patients $>18$ years old admitted in the ICU with (1) a Glasgow Coma Score lower than 8 on presentation, (2) hypoglycemic etiology of coma confirmed or highly likely with at least one measurement of blood glucose concentration $<0.5 \mathrm{~g} / \mathrm{L}$ on presentation, and (3) persistent consciousness disorders after normalizing blood glucose levels defined as persistent Glasgow Coma Score lower than 8 at least up to $24 \mathrm{~h}$ after ICU admission. Patients with possible other causes of consciousness disorders, previous cognitive disorders, hypothermia $<35{ }^{\circ} \mathrm{C}$ or circulatory arrest within $24 \mathrm{~h}$ after ICU admission, or refusal to use their data were excluded.

\section{Data collection}

General data included comorbidities, medical history, clinical findings, prehospital and admission blood glucose levels, Simplified Acute Physiological Score (SAPS II), need for and duration of mechanical ventilation, results of standard electroencephalogram (EEG), brain imaging, evoked potentials if any, main complications and length of ICU stay, decisions to withhold or withdraw care. Morbidity and mortality on ICU discharge were also recorded. The degree of disability was estimated with the modified Rankin Scale (mRS) before hospitalization (value on stable condition before the episode of hypoglycemia), at ICU discharge and, if available, at at least 1 year after ICU discharge [9]. The functional outcome was dichotomized into good (mRS 0-3) and poor (mRS 4-6). Scores range from 0 to 6: 0 indicating no symptoms at all; 1 indicating no significant disability despite symptoms, being able to carry out all usual duties and activities; 2 indicating slight disability, being unable to carry out all previous activities, but able to look after own affairs without assistance; 3 indicating moderate disability, requiring some help, but being able to walk without assistance; 4 indicating moderately severe disability, being unable to walk without assistance and unable to attend to own bodily needs without assistance; 5 indicating severe disability, being bedridden, incontinent and requiring constant nursing care and attention; and 6 indicating death. The mRS before hospitalization and on ICU discharge was evaluated using patients' medical records including daily reports from doctors, nurses and physiotherapists and verified by the patients whenever possible at at least 1-year follow-up phone call. Finally, at least 1-year follow-up mRS was assessed by calling the patient, sometimes with the help of relatives. There was no structured interview, but all investigators were provided with the above-mentioned description of each $\mathrm{mRS}$ value; the reported $\mathrm{mRS}$ was the $\mathrm{mRS}$ of the patient at the time of phone call. Investigators were aware of the entire medical records of the patients they interviewed. To obtain these data, local investigators had to send previous information and consent form to the patient.

\section{Statistical methods}

Descriptive results of continuous variables were expressed as mean and standard deviation or as median and interquartile range, depending on the normality of their distribution. Variables were tested for their association with prognosis by using Pearson's Chi-squared test for categorical data and Mann-Whitney $U$ test for numerical data. A multiple stepwise logistic regression model was established with any covariate with univariate significance of $P$ value $<0.10$ eligible for inclusion in the model. The model was then further calibrated through Hosmer-Lemeshow testing.

\section{Ethical considerations}

The study was approved by the Ethics Committee of the French Language Society of Critical Care Medicine. According to French law on noninterventional and retrospective studies, patients received written information about the study and non-opposition to their participation in the study was sought.

\section{Results}

Among the 15 ICUs invited to participate, one reported that no patient fulfilled the inclusion criteria; nine admitted in the study period at least one patient that 
fulfilled the inclusion criteria and five provided no information. Seventy-three patients were eligible, and a total of 49 patients were finally included (Fig. 1). Their main characteristics are displayed in Table 1. Forty-one patients (84\%) were hospitalized in the ICU within the first $24 \mathrm{~h}$ after the presumed onset of hypoglycemia and the eight remaining patients between 24 and $48 \mathrm{~h}$.
Insulin ( $n=26)$, oral antidiabetic drugs $(n=5)$ or both $(n=1)$ were the cause of hypoglycemia in two-thirds of cases. Among the 46 patients who underwent mechanical ventilation, 10 were not sedated. The most frequent type of sedation was a combination of midazolam and sufentanil for 14 patients. Among other patients, 9 were sedated by propofol, 7 by the association of midazolam

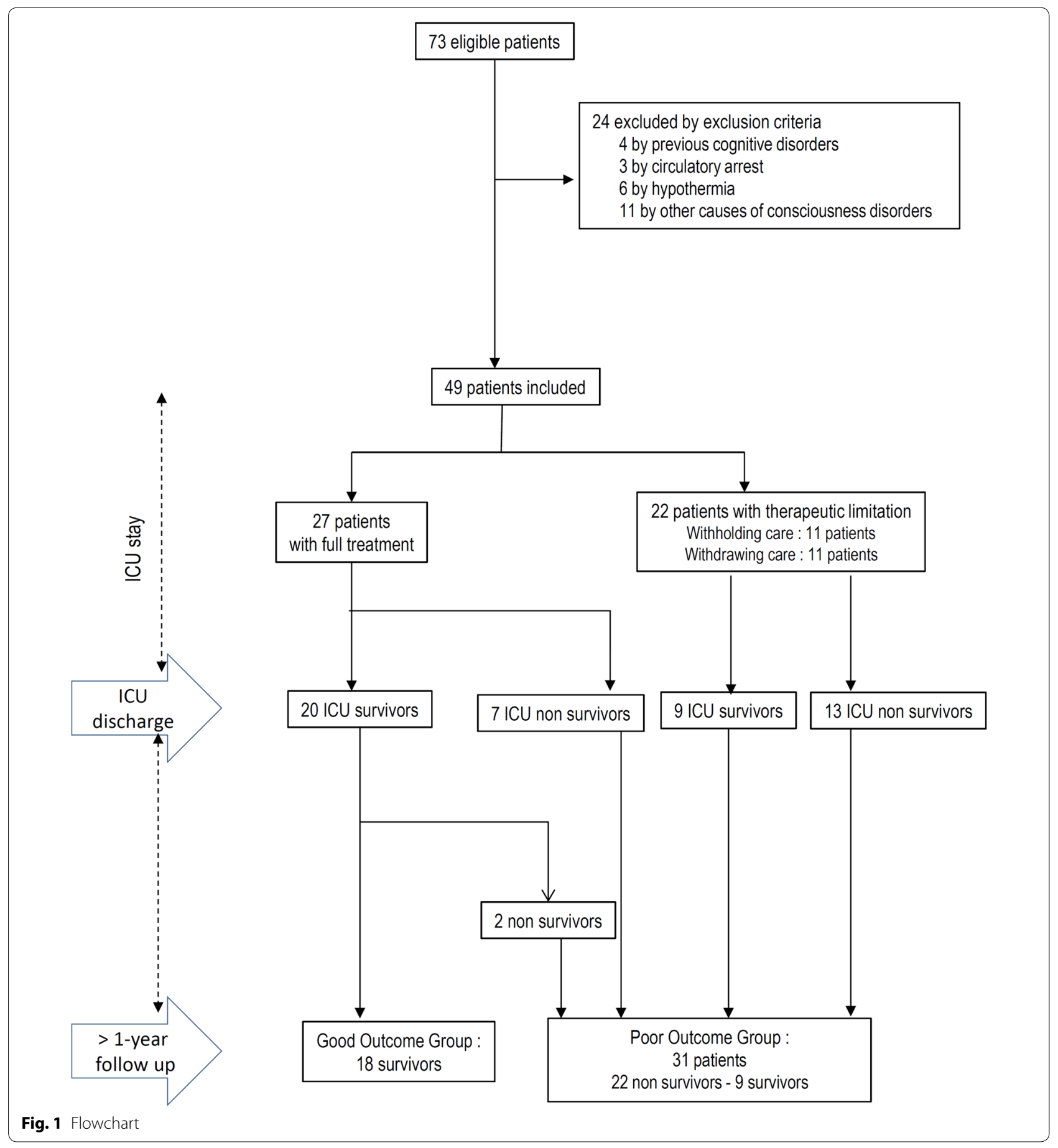


Table 1 Baseline characteristics and outcome of patients with hypoglycemic encephalopathy

\begin{tabular}{|c|c|c|c|c|}
\hline & $\begin{array}{l}\text { All } \\
(n=49)\end{array}$ & $\begin{array}{l}\text { Good outcome } \\
(\mathrm{mRS} \leq 3) \\
(n=18)\end{array}$ & $\begin{array}{l}\text { Poor outcome }(\mathrm{mRS}>3) \\
(n=31)\end{array}$ & $P$ value \\
\hline Male & $27(55)$ & $9(50)$ & $18(58)$ & 0.28 \\
\hline Age (years) & $55[44-70]$ & $52[39-64]$ & $56[45-71]$ & 0.11 \\
\hline Chronic alcohol abuse & $17(35)$ & $5(28)$ & $12(39)$ & 0.23 \\
\hline Diabetes & $29(59)$ & $8(44)$ & $21(68)$ & 0.01 \\
\hline SAPS $\|$ & $55[49-60]$ & $54[44-61]$ & $55[50-60]$ & 0.75 \\
\hline mRS prior to ICU admission & $1[0-2]$ & $0[0-1]$ & $1[0-2]$ & 0.03 \\
\hline \multicolumn{5}{|l|}{ Etiology } \\
\hline Insulin and/or oral antidiabetics & $32(65)$ & $12(67)$ & $20(65)$ & 0.89 \\
\hline Neuroendocrine carcinoma & $8(16)$ & $2(11)$ & $6(19)$ & \\
\hline Alcohol abuse & $2(4)$ & $1(6)$ & $1(3)$ & \\
\hline Adrenal insufficiency & $1(2)$ & $1(6)$ & $0(0)$ & \\
\hline Unknown & $6(12)$ & $2(11)$ & $4(13)$ & \\
\hline Duration of hypoglycemia, $\min (n=27)$ & $480[35-720]$ & $45[30-120]$ & 660 [187-1283] & 0.006 \\
\hline Initial glycemia (g/L) & $0.21 \pm 0.02$ & $0.22 \pm 0.03$ & $0.20 \pm 0.02$ & 0.51 \\
\hline Glycemia on ICU admission (g/L) & $1.01 \pm 0.58$ & $1.05 \pm 0.16$ & $1.50 \pm 0.44$ & 0.45 \\
\hline \multicolumn{5}{|l|}{ GCS } \\
\hline Initial & $4 \pm 1$ & $4 \pm 1$ & $4 \pm 1$ & 0.45 \\
\hline After glycemia normalization & $5 \pm 1$ & $5 \pm 2$ & $5 \pm 1$ & 0.89 \\
\hline Seizures & $8(16)$ & $6(33)$ & $2(6)$ & $<0.001$ \\
\hline \multicolumn{5}{|l|}{ Temperature $\left({ }^{\circ} \mathrm{C}\right)$} \\
\hline Initial & $36.6 \pm 0.2$ & $36.7 \pm 1.2$ & $36.7 \pm 0.5$ & 0.91 \\
\hline Highest during first $24 \mathrm{~h}$ & $37.9 \pm 0.8$ & $37.7 \pm 0.8$ & $38.0 \pm 1.2$ & 0.36 \\
\hline Initial arterial pH & $7.38 \pm 0.08$ & $7.36 \pm 0.12$ & $7.40 \pm 0.06$ & 0.23 \\
\hline Initial lactate level (mmol/L) & $2.54 \pm 0.67$ & $3.23 \pm 1.36$ & $2.10 \pm 1.75$ & 0.17 \\
\hline Time to final glycemia normalization (min) & $180[60-705]$ & $480[120-720]$ & $180[67-630]$ & 0.78 \\
\hline Mechanical ventilation & $46(94)$ & $16(89)$ & $30(97)$ & 0.55 \\
\hline Length of mechanical ventilation (days) & $7[3-12]$ & $4.5[2-8]$ & $8[6-13]$ & 0.08 \\
\hline Normal EEG $(n=34)$ & $1(3)$ & $1(10)$ & $0(0)$ & 0.37 \\
\hline Normal brain imaging $(n=45)$ & $25(55)$ & $11(73)$ & $14(46)$ & 0.009 \\
\hline \multicolumn{5}{|l|}{ Acquired ICU complications } \\
\hline Pneumonia & $25(51)$ & $8(44)$ & $17(55)$ & 0.28 \\
\hline Septic shock & $8(16)$ & $3(17)$ & $5(16)$ & 0.78 \\
\hline ICU length of stay (days) & $11[5.5-17]$ & $8[4-14]$ & $11[7-17]$ & 0.57 \\
\hline At least 1-year follow-up, days ( $n=27$ ) & $840[394-1768]$ & $826[407-1906]$ & 1373 [388-1997] & 0.93 \\
\hline
\end{tabular}

Values are $n(\%)$, mean \pm SD or median [IQR]

GCS Glasgow Coma Score, SAPS I/ Simplified Acute Physiological Score (version II)

and fentanyl and 6 by midazolam only. Upon ICU admission, 20 patients $(41 \%)$ were still hypoglycemic (glycemia $<0.7 \mathrm{~g} / \mathrm{L})$.

Forty-five patients (92\%) underwent brain imaging, 20 and 25 of them using MRI and CT scan, respectively. Most brain imaging examinations $(n=24)$ were performed on ICU admission with a median time of 0 day [0-5.5] (Table 2). Imaging was considered as normal in 25 patients. However, normal brain CT scans were significantly more frequent than normal MRIs $(P<0.001)$.
In the 20 patients with abnormal imaging, lesions were bilateral in 16 of them. The most frequent abnormalities were located in the cortex (17\%), the basal ganglia (15\%) and the white matter (15\%).

A standard EEG was performed in 34 patients (69\%). Only one patient displayed a normal examination. The most frequently reported abnormality was a slowed brain electrical activity (22 out of 25 patients in the poor outcome group and 6 out of 9 in the poor outcome group) $(P=0.41)$. 
Table 2 Type and results of imaging

\begin{tabular}{|c|c|c|c|c|}
\hline & All $(n=49)$ & $\begin{array}{l}\text { Good outcome } \\
(\mathrm{mRS} \leq 3) \\
(n=18)\end{array}$ & $\begin{array}{l}\text { Poor outcome } \\
(\mathrm{mRS}>3) \\
(n=31)\end{array}$ & $P$ value \\
\hline CTscan & 25 & 10 & 15 & 0.15 \\
\hline \multicolumn{5}{|c|}{ Localization or type of lesions } \\
\hline No lesion & & 10 & 13 & 0.5 \\
\hline Unilateral & & 0 & 1 & 1.0 \\
\hline Bilateral & & 0 & 1 & 1.0 \\
\hline Cortex & & 0 & 0 & 1.0 \\
\hline $\begin{array}{l}\text { Basal } \\
\text { ganglia }\end{array}$ & & 0 & 0 & 1.0 \\
\hline $\begin{array}{l}\text { White } \\
\text { matter }\end{array}$ & & 0 & 0 & 1.0 \\
\hline Ischemia & & 0 & 1 & 1.0 \\
\hline Edema & & 0 & 1 & 1.0 \\
\hline$M R I$ & 20 & 5 & 15 & 0.15 \\
\hline \multicolumn{5}{|c|}{ Localization or type of lesions } \\
\hline No lesion & & 1 & 1 & 0.45 \\
\hline Unilateral & & 1 & 2 & 0.55 \\
\hline Bilateral & & 3 & 12 & 0.55 \\
\hline Cortex & & 2 & 7 & 1.0 \\
\hline $\begin{array}{l}\text { Basal } \\
\text { ganglia }\end{array}$ & & 2 & 6 & 1.0 \\
\hline $\begin{array}{l}\text { White } \\
\text { matter }\end{array}$ & & 2 & 6 & 1.0 \\
\hline Ischemia & & 1 & 4 & 1.0 \\
\hline Edema & & 0 & 2 & 1.0 \\
\hline No imaging & 4 & 3 & 1 & 0.15 \\
\hline
\end{tabular}

Data expressed in numbers

$C T$ computed tomography, MRI magnetic resonance imaging

Withholding life-sustaining care was decided for 11 patients (22\%) after a median ICU stay of 7 [6-13] days, and withdrawing care was secondarily decided for two of them. Among the remaining patients, withdrawing care was decided as a first decision for 11 patients $(22 \%)$ after a median ICU stay of 11 [6-15] days. No decision of therapeutic limitation was observed in the patients who reached a good outcome. Motivations included no hope for improvement $(n=19)$, expected limited quality of life $(n=8)$, expected limited autonomy $(n=6)$, heaviness of comorbidities and medical history $(n=5)$, severity of ICU complications $(n=3)$ and family request $(n=2)$. Nine patients who underwent care limitation, including three patients with withdrawal of care, survived to ICU discharge, all with a final poor outcome. The characteristics of patients with a decision of therapeutic limitation are compared to patients with no therapeutic restriction in Table 3 . They displayed a significantly longer time under mechanical ventilation $(P=0.002)$ and ICU length of stay $(P=0.007)$ than patients with no therapeutic limitation.
Twenty patients (41\%) died during ICU stay at a median time of 10 days [7-16] after admission, in relation to therapeutic limitation $(n=13)$, respiratory distress $(n=3)$, sudden cardiac arrest $(n=2)$ and brain death $(n=2)$.

As shown in Fig. 2, there was a marked decline in functional status between admission and ICU discharge with a median increase in mRS of 4 [2-5]. Among survivors with poor outcome at ICU discharge $(n=15)$, one died 10 months later, six improved their outcome at at least 1-year follow-up with a median decrease in mRS of $-2[-2.5$ to -1.75$]$ including five patients who finally reached the good outcome group, and eight remained unchanged. Among the patients with good outcome at ICU discharge $(n=14)$, one died, seven further improved their outcome at 1 year with a median decrease in $\mathrm{mRS}$ of -2 [ -2.0 to -1.0$]$, whereas one patient deteriorated while remaining in the good outcome group (from mRS 1 to $\mathrm{mRS} 2$ ) and six remained unchanged. Finally, at 1-year follow-up, 18 patients (37\%) displayed a good outcome with a median decrease in $\mathrm{mRS}$ of -1.5 [ -2 to 0$]$ as compared with ICU discharge.

Univariate analysis (Table 1) showed that diabetes, low $\mathrm{mRS}$ prior to ICU admission, short duration of hypoglycemia, onset of seizures and a normal brain imaging were associated with a better 1-year outcome. The topography of the lesions did not display any prognostic value.

On multivariate analysis, only low mRS prior to ICU admission (OR 2.6; 95\% CI 1.1-6.3; $P=0.03$ ) and normal brain imaging (OR 7.1; 95\% CI 1.1-44; $P=0.03$ ) remained significantly predictive of outcome. The duration of hypoglycemia was not entered in the statistical analysis since values were unknown in 22 patients. However, as displayed in Fig. 3, all patients who remained hypoglycemic for $480 \mathrm{~min}$ or more evolved poorly while seven out of 12 patients with an exposition time to hypoglycemia below $300 \mathrm{~min}$ had a good outcome $(P<0.006)$.

\section{Discussion}

In this multicenter study, we identified 49 patients hospitalized in several ICUs for a prolonged hypoglycemic encephalopathy over 10 years. A poor prognosis was observed in $31(63 \%)$ patients including 20 ICU non-survivors, two follow-up non-survivors and nine survivors (Fig. 1). Noteworthy, six patients who were discharged from the ICU with poor functional status improved their $\mathrm{mRS}$ at 1-year follow-up, including five who reached the good outcome group. Seeking to identify predictive variables of long-term outcome useful in daily practice, we found that low mRS prior to ICU admission and the absence of abnormalities on first brain imaging were significantly, although not strongly, predictive of outcome. 
Table 3 Characteristics of patients with or without a decision of care limitation

\begin{tabular}{|c|c|c|c|}
\hline & $\begin{array}{l}\text { No care limitation } \\
(n=27)\end{array}$ & $\begin{array}{l}\text { Withholding/withdrawing care } \\
(n=22)\end{array}$ & $P$ value \\
\hline Male & $13(48)$ & $14(64)$ & 0.38 \\
\hline Age (years) & $53[42-64]$ & 58 [49-72] & 0.15 \\
\hline Chronic alcohol abuse & $20(74)$ & $9(41)$ & 0.04 \\
\hline Diabetes & $14(52)$ & $15(68)$ & 0.38 \\
\hline SAPS $\|$ & $53[47-60]$ & $57[51-63]$ & 0.17 \\
\hline mRS prior to ICU admission & $1[0-1]$ & $1[0-2]$ & 0.40 \\
\hline Duration of hypoglycemia, $\min (n=27)$ & $90[31-525]$ & 720 [480-810] & 0.07 \\
\hline Initial glycemia (g/L) & $0.20 \pm 0.13$ & $0.22 \pm 0.12$ & 0.45 \\
\hline Glycemia on ICU admission (g/L) & $1.42 \pm 1.49$ & $1.24 \pm 1.18$ & 0.86 \\
\hline \multicolumn{4}{|l|}{ GCS } \\
\hline Initial & $4 \pm 1$ & $4 \pm 1$ & 0.06 \\
\hline After glycemia normalization & $5 \pm 2$ & $5 \pm 1$ & 0.55 \\
\hline Seizures & $7(26)$ & $1(4)$ & 0.04 \\
\hline \multicolumn{4}{|l|}{ Temperature $\left({ }^{\circ} \mathrm{C}\right)$} \\
\hline Initial & $36.5 \pm 1.2$ & $36.9 \pm 1.5$ & 0.49 \\
\hline Highest during first $24 \mathrm{~h}$ & $37.8 \pm 1.2$ & $38.1 \pm 1.4$ & 0.28 \\
\hline Initial arterial pH & $7.36 \pm 0.14$ & $7.41 \pm 0.09$ & 0.39 \\
\hline Initial lactate level (mmol/L) & $3.05 \pm 3.04$ & $1.81 \pm 1.09$ & 0.18 \\
\hline Time to final glycemia normalization (min) & $390[112-720]$ & $180[67-480]$ & 0.42 \\
\hline Mechanical ventilation & $24(89)$ & $22(100)$ & 0.24 \\
\hline Length of mechanical ventilation (days) & $5.5[2-8]$ & $11[7-16]$ & 0.002 \\
\hline Normal EEG $(n=34)$ & $1(3.7)$ & $0(0)$ & 0.41 \\
\hline Normal brain imaging $(n=45)$ & $17(63)$ & $8(36)$ & 0.04 \\
\hline \multicolumn{4}{|l|}{ Acquired ICU complications } \\
\hline Pneumonia & $12(44)$ & $13(59)$ & 0.39 \\
\hline Septic shock & $5(19)$ & $3(14)$ & 0.72 \\
\hline ICU length of stay (days) & $7[4-13]$ & $14[9-24]$ & 0.007 \\
\hline
\end{tabular}

Values are $n(\%)$, mean \pm SD or median [IQR]

GCS Glasgow Coma Score, SAPS I/ Simplified Acute Physiological Score (version II)

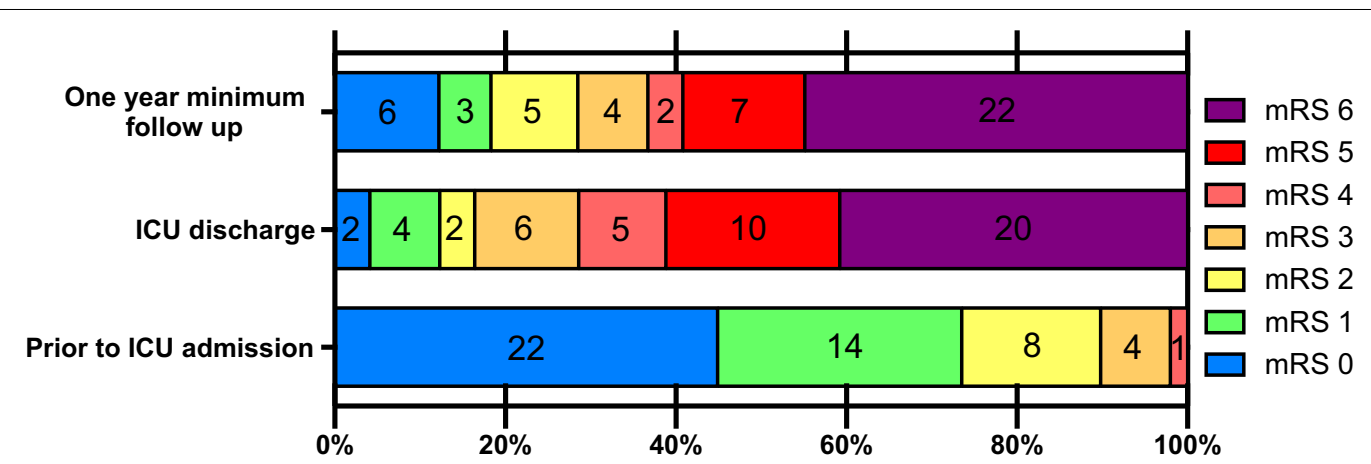

Fig. 2 Distributions of patients modified Rankin Scale (mRS) prior to ICU admission, at ICU discharge and at a minimum of 1-year follow-up. mRS prior to admission was the mRS on stable condition before the episode of hypoglycemia. mRS 0 indicates no symptoms at all and mRS 6 indicates death. Good outcome is defined by mRS 0-3 and poor outcome by mRS 4-6 


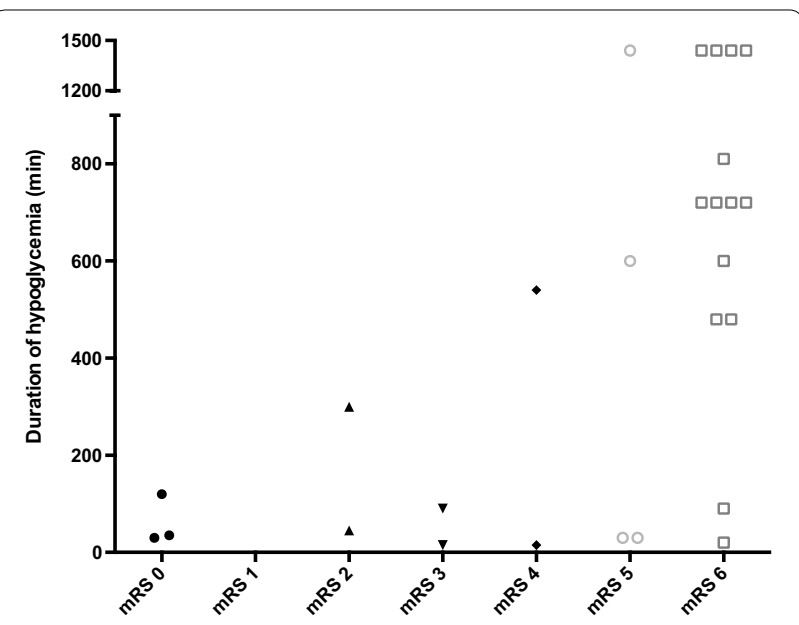

Fig. 3 Modified Rankin Scale (mRS) at a minimum of 1-year follow-up according to the duration of hypoglycemia. Data were lacking for 22 patients

Finally, the outcome of all patients with a long hypoglycemic duration was poor.

Available published data on prognosticators in prolonged hypoglycemic encephalopathy are based on cases series with a limited number of patients. To our knowledge, this study has the largest population of prolonged hypoglycemic encephalopathy requiring ICU hospitalization. Ikeda et al. [2] published a retrospective study of 165 patients to find predictors of 1-week good outcome in hypoglycemic encephalopathy. They included also patients with "hypoglycemic consciousness disturbance" which regained consciousness immediately after glucose administration, while our population was only constituted of severe patients requiring ICU because of a persistent coma over $24 \mathrm{~h}$. Furthermore, 1-week follow-up was probably too short to fully evaluate this pathology. The retrospective study by Witsch et al. [1] included 15 patients from three centers with similar overall population's characteristics regarding age, sex ratio, initial blood glucose levels, clinical severity and etiology than ours. Six of them died, two were lost to follow-up, five had a longterm good outcome and two had a poor outcome. These outcomes are consistent with the findings of the present study. However, the series was too small to identify outcome predictive factors.

As already known, hypoglycemic encephalopathy is more common in diabetic patients and diabetes was associated with poor outcome in univariate, but no longer in multivariate analysis. These data are consistent with findings of previous studies [1, 2, 7, 8]. They may be explained by a defective glucose counter-regulation in type-1 diabetic patients caused by frequent hypoglycemic episodes called "hypoglycemia-associated autonomic failure." Symptoms due to the lack of glucose appear later and predispose to hypoglycemic encephalopathy [10].

Duration of hypoglycemia was a significant predictor of outcome in univariate analysis, but was not included in the multivariate analysis due to several missing data. For many patients, time between hypoglycemia onset and first glucose administration was impossible to precisely assess. This length was only available in 27 (55\%) patients. Interestingly, a prolonged hypoglycemia was significantly associated with poor outcome, so that in our study, all patients who underwent hypoglycemia for at least $480 \mathrm{~min}$ had a poor outcome at 1-year follow-up $(n=15$; see Fig. 3$)$. In the paper by Ikeda et al. [2], a similar relationship was observed with a cutoff time for no good prognosis of about $48 \mathrm{~h}$ of hypoglycemia, which is longer than in the present study. These differences may be explained by both different criteria of good outcome and a very earlier outcome assessment than in the present study. Nevertheless, both studies strongly suggest that duration of hypoglycemia should be a major determinant of outcome.

Occurrence of seizures, most of them being observed early, was identified as a factor of good prognosis in univariate analysis. This issue has not been studied in the previous main hypoglycemic encephalopathy series $[1$, 2]. Our findings are not consistent with the results of a recent rodent hypoglycemic model study, showing that seizures and especially their frequency were associated with increased mortality [11]. Interestingly, a retrospective study about 425 patients with subarachnoid hemorrhage found that patients who presented early-onset seizures had a final good outcome, despite initial poor $\mathrm{mRS}$ [12]. Whatever the explanation of our findings, early-onset seizures should not be considered at least as adding more clinical severity in prolonged hypoglycemic encephalopathy.

Brain imaging has been evaluated as a prognosis tool [6-8, 13-16]. Yanagawa et al. [13] reported a case explaining how MRI had been useful to predict awakening and sequelae of their patient who underwent hypoglycemic encephalopathy. Johkura et al. [6] prospectively studied early diffusion MRI as a predictor of short-term outcome in 36 patients with hypoglycemic encephalopathy. The absence of lesions on the first early diffusion MRI was associated with good outcome. Conversely, Witsch et al. [1] did not find any prognostic value in imaging, but this retrospective study recruited 15 patients, of them only three had MRI. In the present study, lesion localization at first imaging (including MRI and CT scan) was not predictive of outcome but normal early imaging that was mostly observed in brain CT scans was considered as a factor of good outcome. 
In contrast to Ikeda et al. [2], the initial serum glucose concentration, the body temperature during hypoglycemia and the blood lactate concentration were not related to the final patient outcome in our study. This discrepancy may be explained by several reasons. Ikeda et al. [2] defined good and poor outcomes groups using Glasgow Outcome Scale (GOS) and poor outcome included patients with GOS from 1 to 4 . Thereby, patients with moderate disability but independent in daily living were considered as poor outcome patients, while they would have been included in our good outcome group. Furthermore, even if Ikeda et al. [2] included 165 patients, lactate measurement was available in only 19 of them.

Therapeutic limitation was decided in 22 patients. All of them further displayed a poor outcome. Decisions were made in seven patients within 1 week after ICU admission, with a minimal time of 2 days $(n=1)$. Decisions of therapeutic limitation were mostly based on the clinical severity of neurological insult and the expected absence of significant improvement with time. A similar reasoning is usual in anoxic-hypoxic encephalopathy. However, mechanisms of brain damage are different $[17,18]$, so that clinical prognostic tools used for decades in hypoxic encephalopathy have yet to be validated in hypoglycemic encephalopathy. Keeping in mind that available evidence about prognosis of severe hypoglycemic encephalopathy is scarce, therapeutic limitation could constitute an important bias, leading to "self-fulfilling prophecies" [19]. On the one hand, patients with therapeutic limitations had a longer time under mechanical ventilation and stay in the ICU, a longer duration of hypoglycemia, and survivors did not improve their outcome over time as compared with not limited patients. On the other hand, the expected time for clinical improvement appears to be long as we observed that five patients with no therapeutic limitation discharged from the ICU with mRS > 3 improved enough to be later in the good outcome group. Taken together, these findings suggest that decisions to withhold or withdraw care should be taken very cautiously, obviously not prematurely after ICU admission. Ten to fifteen days with no clinical improvement could be a minimal threshold, based on the minimal time from admission to decision of therapeutic limitation among the patients who survived their ICU stay. In addition, normal brain imaging, especially using RMI, could help to further postpone a decision to limit care.

Our study has several limitations. Several data such as duration of hypoglycemia were missing. Selection biases cannot be excluded regarding the non-responding invited ICU centers. Furthermore, initial glycemia was mostly assessed (94\%) using capillary glucometers that are less reliable than laboratory measurements using blood venous samples. Our population was small due to the scarcity of this disease, and a larger population would have allowed a more robust multivariate analysis. In addition, several patients did not undergo brain MRI, a more sensitive tool than CT scan, and four of them had no imaging at all. Continuous EEG monitoring, never performed here could have been more relevant to rule out subclinical status epilepticus or better delineate patterns with prognosis value. Finally, mRS assessment was not based on a standardized questionnaire, which could have led to possible mistakes.

\section{Conclusion}

This multicenter study confirms that prolonged hypoglycemic encephalopathy is a severe condition leading to a poor long-term outcome. Factors of good outcome were a low mRS prior to admission and normal brain imaging, while the hypothesis of a long duration of hypoglycemia as a predictor of poor outcome is suggested. However, some patients with severe impairment at ICU discharge may improve later, suggesting that therapeutic limitation should not be decided early. Finally, the present findings offer prognostic tools, which need to be further delineated in larger, prospective investigations.

\section{Abbreviations}

CT: computed tomography; EEG: electroencephalogram; GOS: Glasgow Outcome Scale; ICU: intensive care unit; MRI: magnetic resonance imaging; mRS: modified Rankin Scale; SAPSII: Simplified Acute Physiological Score (version II).

\section{Authors' contributions}

GB was involved in conception and design, data acquisition, analysis and interpretation of the data, and drafting the manuscript. BM was involved in conception and design, data acquisition, and drafting the manuscript. LA, $\mathrm{GL}, \mathrm{NL}, \mathrm{FS}, \mathrm{SGa}, \mathrm{NB}, \mathrm{AJ}, \mathrm{HO}$ were involved in conception and design and data acquisition. SGi was involved in statistical analysis, analysis and interpretation of the data, and drafting the manuscript. PEB was involved in conception and design, data acquisition, analysis and interpretation of the data, and drafting the manuscript. All authors had full access to the data, take responsibility for the integrity of the data and the accuracy of the analysis. All authors read and approved the final manuscript.

\footnotetext{
Author details

${ }^{1}$ Service de Réanimation médicale, Hôpital Central, CHU de Nancy, Av. de Lattre de Tassigny, 54035 Nancy Cedex, France. ${ }^{2}$ Service de Réanimation Médicale et Toxicologique, CHU Lariboisière, INSERM U1144, Université Paris Diderot, Paris, France. ${ }^{3}$ Service de Réanimation Médicale, Hôpital Edouard Herriot, Lyon, France. ${ }^{4}$ Service de Réanimation Polyvalente, CHR de Metz-Thionville, Metz, France. ${ }^{5}$ Service de Réanimation Médicale et de Médecine Hyperbare, CHU et Université d'Angers, Angers, France. ${ }^{6}$ Service de Réanimation Médicale, Hôpital de Hautepierre, HUS, Fédération de Médecine Translationnelle de Strasbourg et U1121 INSERM, Université de Strasbourg, Strasbourg, France. ${ }^{7}$ Service de Réanimation Médico-Chirurgicale, Hôpital Louis Mourier, Colombes, France. ${ }^{8}$ UMRS 1123, Univ Paris Diderot, Paris, France. ${ }^{9}$ Service de Réanimation Polyvalente, CH de St Brieuc, St Brieuc, France. ${ }^{10}$ Service de Réanimation Médicale, CHU de Poitiers, Poitiers, France. ${ }^{11}$ Service de Réanimation médico-chirurgicale, $\mathrm{CH}$ de Poissy-Saint Germain en Laye, Poissy, France.
}

\section{Acknowledgements}

None. 


\section{Competing interests}

The authors declare that they have no competing interests.

\section{Availability of data and materials section}

The datasets used and/or analyzed during the current study were available from the corresponding author on reasonable request.

\section{Ethics approval and consent to participate}

The study was approved by the Ethics Committee of the French Language Society of Critical Care Medicine (Number SRLF-CE 15-22). According to French law on noninterventional and retrospective studies, patients received written information about the study and non-opposition to their participation in the study was sought.

\section{Publisher's Note}

Springer Nature remains neutral with regard to jurisdictional claims in published maps and institutional affiliations.

Received: 4 November 2016 Accepted: 8 May 2017

Published online: 22 May 2017

\section{References}

1. Witsch J, Neugebauer H, Flechsenhar J, Jüttler E. Hypoglycemic encephalopathy: a case series and literature review on outcome determination. J Neurol. 2012:259:2172-81.

2. Ikeda T, Takahashi T, Sato A, et al. Predictors of outcome in hypoglycemic encephalopathy. Diabetes Res Clin Pract. 2013;101:159-63.

3. Cryer PE. Mechanisms of sympathoadrenal failure and hypoglycemia in diabetes. J Clin Invest. 2006:116:1470-3.

4. Group IHS. Minimizing hypoglycemia in diabetes. Diabetes Care. 2015:38:1583-91.

5. Yokoi S, Yasui K, Hasegawa Y, Kasai T, Inagaki A, Sobue G. A case of hypoglycemic coma with good outcome despite sustained unconsciousness and widespread leukoencephalopathy. Rinshō Shinkeigaku Clin Neurol. 2013:53:724-7.
6. Johkura K, Nakae Y, Kudo Y, Yoshida TN, Kuroiwa Y. Early diffusion MR imaging findings and short-term outcome in comatose patients with hypoglycemia. Am J Neuroradiol. 2012;33:904-9.

7. Ma J-H, Kim Y-J, Yoo W-J, et al. MR imaging of hypoglycemic encephalopathy: lesion distribution and prognosis prediction by diffusion-weighted imaging. Neuroradiology. 2009;51:641-9.

8. Kang EG, Jeon SJ, Choi SS, Song CJ, Yu IK. Diffusion MR imaging of hypoglycemic encephalopathy. Am J Neuroradiol. 2010;31:559-64.

9. Bonita R, Beaglehole R. Recovery of motor function after stroke. Stroke. 1988;19:1497-500.

10. Terpstra M, Moheet A, Kumar A, Eberly LE, Seaquist E, Öz G. Changes in human brain glutamate concentration during hypoglycemia: insights into cerebral adaptations in hypoglycemia-associated autonomic failure in type 1 diabetes. J Cereb Blood Flow Metab. 2014;34:876-82.

11. Maheandiran M, Mylvaganam S, Wu C, et al. Severe hypoglycemia in a juvenile diabetic rat model: presence and severity of seizures are associated with mortality. PLOS ONE. 2013;8(12):e83168.

12. Fung $C$, Balmer $M$, Murek $M$, et al. Impact of early-onset seizures on grading and outcome in patients with subarachnoid hemorrhage. J Neurosurg. 2015:122:408-13.

13. Yanagawa Y, Isoi N, Tokumaru AM, Sakamoto T, Okada Y. Diffusionweighted MRI predicts prognosis in severe hypoglycemic encephalopathy. J Clin Neurosci. 2006;13:696-9.

14. Lo L, Tan CHA, Umapathi T, Lim CC. Diffusion-weighted MR imaging in early diagnosis and prognosis of hypoglycemia. Am J Neuroradiol. 2006:27:1222-4.

15. Chan R, Erbay S, Oljeski S, Thaler D, Bhadelia R. Case report: hypoglycemia and diffusion-weighted imaging. J Comput Assist Tomogr. 2003;27:420-3.

16. Ohshita T, Imamura E, Nomura E, Wakabayashi S, Kajikawa H, Matsumoto M. Hypoglycemia with focal neurological signs as stroke mimic: clinical and neuroradiological characteristics. J Neurol Sci. 2015;353:98-101.

17. Auer RN, Hugh J, Cosgrove E, Curry B. Neuropathologic findings in three cases of profound hypoglycemia. Clin Neuropathol. 1989:8:63-8.

18. Auer RN. Hypoglycemic brain damage. Metab Brain Dis. 2004;146:169-75.

19. Becker KJ, Baxter AB, Cohen WA, et al. Withdrawal of support in intrac erebral hemorrhage may lead to self-fulfilling prophecies. Neurology. 2001;56:766-72.

\section{Submit your manuscript to a SpringerOpen ${ }^{\odot}$ journal and benefit from:}

- Convenient online submission

- Rigorous peer review

- Immediate publication on acceptance

- Open access: articles freely available online

- High visibility within the field

- Retaining the copyright to your article 